\title{
COMPUTER AIDED FREQUENCY PLANNING FOR THE RADIO AND TV BROADCASTS
}

\author{
A. Altintaş, O. Ocalı, S. Topçu, S. G. Tanyer*, and H. Köymen \\ Bilkent University, Department of Electrical and Electronics Eng., Bilkent, 06533, Ankara, Turkey, \\ *Ankara University, Department of Electronics Eng., Tandoğan, 06100, Ankara, Turkey.
}

\begin{abstract}
The frequency planning of the VHF and UHF broadcasts in Turkey is described. This planning is done with the aid of computer databases and digital terrain map. The frequency offset is applied whenever applicable to increase the channel capacity. The offset assignment is done through Simulated Annealing algorithm. The international rules and regulations concerning Turkey are also considered.
\end{abstract}

\section{Introduction}

The introduction of terrestrial VHF and UHF broadcast is not new. However, especially in many developing countries, this broadcast has been under the monopoly of the government. Only recently, the private broadcasting became a reality, even though in a de facto, and disorganized manner in many occasions. This brought up the need to have a nationwide re-planning, allocation, and management of the broadcast frequencies. The pressure from the public as well as the international agreements require that the planning has to be done in the shortest possible time with realistic and applicable goals. Therefore, an integrated approach with maximum utilization of computers and relevant databases has to be followed.

For the planning of Turkish broadcast spectrum, we have used five main computer tools and databases. The first one is a propagation simulation software which models the propagation environment and calculates the attenuation of the field strength over a given terrain. The second one is the geographical database of the planning region. This includes a digital terrain map of the country. The third is the demographical database. The forth is the list of all present transmitters and their electronic and geographical parameters. The last one is the international rules and agreements concerning the region. All of these tools are either acquired or produced and they have been properly integrated to have an optimum frequency allocation.

Publisher Item Identifier: S 0018-9316(96)07166-1
The main purpose is to maximize the interference free coverage area for each transmitter. To this end, for each transmitter propagation simulation studies are obtained. From these studies, channel assignment can be done by considering the international coordination agreements. However, for TV broadcasts a better option is to use frequency offsets to get the maximum number of available channels. This is done using a Simulated Annealing algorithm. In [1], the Simulated Annealing algorithm is used for the efficient frequency assignment, but for a different problem. The flowchart of the approach is summarized in Fig.(1).

In addition to the channel assignment plan, the population in the coverage area and the services provided to any town in the country is determined. This information is used for the licencing fees to be charged to the stations and also for the classification of broadcasters into the national, regional, or local categories.

\section{Tools of Planning}

The necessary ingredient of a computer aided frequency planning is the prediction of field strength variation over the terrain due to a given transmitter. This requires a propagation simulation software which utilizes the topography of the terrain. Fortunately, different commercial software from various sources are available for this purpose. The next step is to decide on the propagation models to be used. At present, a number of different propagation models are available and the comparative advantages of these models are still under investigation [2]. The most widely known models are prepared by the CCIR of ITU [3] and the FCC of US Government (part 73, broadcast). These models are based on intensive experimental measurements in the VHF and UHF bands. The terrain effect on the propagation is included in these models only statistically. The actual topografic structure may cause strong reflections and diffractions which can also be added to these models. For the coverage area studies, we

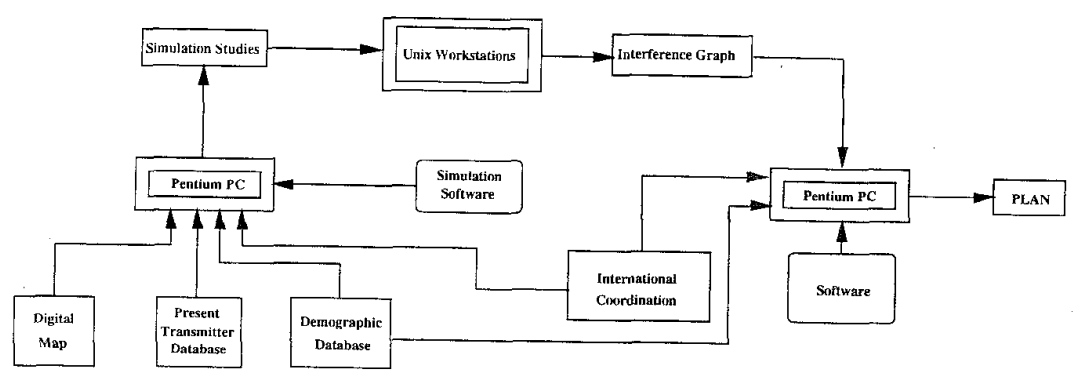

Figure 1. The flowchart of the frequency planning. 


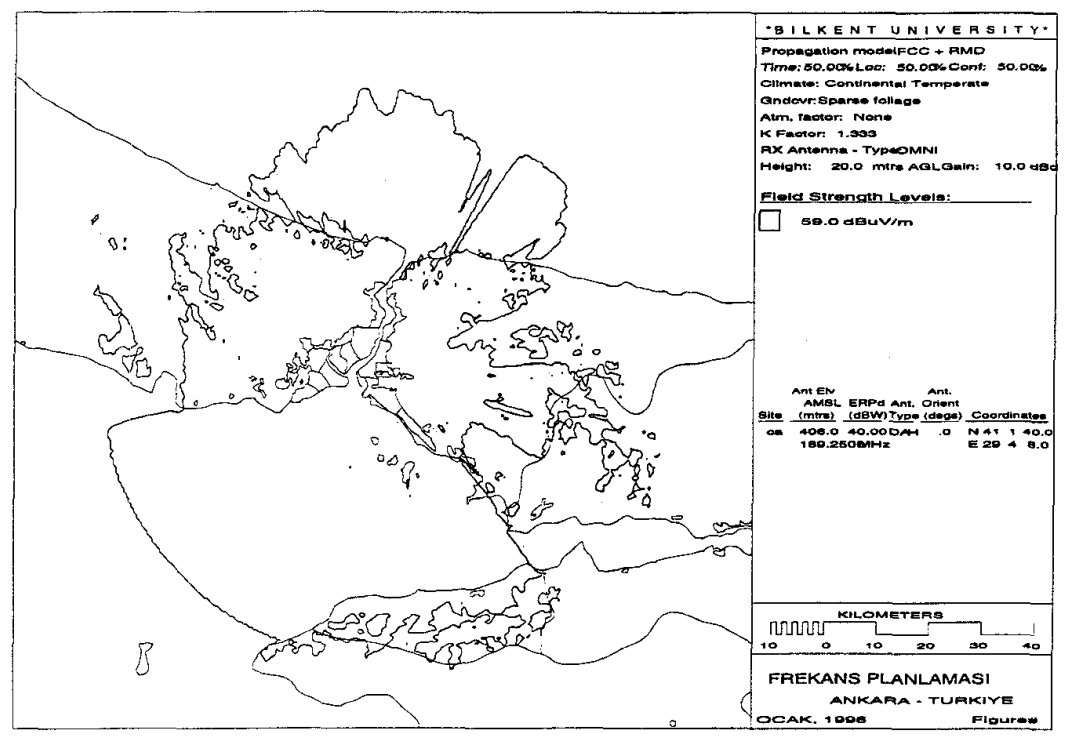

Figure 2. Coverage area contour plot of the Camlica (istanbul) station in the VHF.

have accepted and used such a model based on FCC measurements and augmented by reflection and diffraction contributions of the terrain. As is well known, CCIR attenuation curves begin at $10 \mathrm{~km}$. away from the transmitter, making it unsuitable for coverage estimates. We have made some measuments in Ankara and in Istanbul, and the measured field strengths were within 10 $\mathrm{dB}$ of the computer generated data obtained through the adopted propagation model.

Around the transmitter, we can. define an area, in which the field strength is strong enough to give a satisfactory grade of sound or picture quality. We can call this area as "the coverage area". Similarly, "co-channel and adjacent channel interference areas" are defined such that in these areas either the same or the adjacent channels may not be used by any other transmitter. To be able to transmit the same or adjacent channels, the relevant protection ratios between the desired and undesired (interference) field strengths have to be satisfied. These protection ratios are listed in the EBU [4]-[5] publications. A sample TV coverage area contour plot is given in Fig.(2) for a TV transmission station in the VHF band in İstanbul. A better insight is gained through a gray scale plotting of the field strength around the transmitter. Fig.(3) shows such a plot for the same station around Istanbul. Here it is easier to identify the effect of the topography on the field strength distributions. It is noted that the field strength values are calculated over radial lines centered at the transmitter location. This analysis is valid when the reflection and diffractions from the vertical discontinuities are small. Fortunately, most natural topographic boundaries are not vertical as opposed to man made buildings. So, the limitation to radial lines (2-D) is expected to work well especially outside highly populated urban areas [6].

The next step is to decide the transmitter locations, transmitter powers and antenna characteristics. In practice, this is usually done by the demand of the public. Therefore, the coverage starts from densely to sparsely populated areas. The same logic is applied here and the aim is set to cover all cities and towns over a threshold population. Also, all the information about the trans- mitter locations of the existing broadcasters are collected into a database, and this database is consulted whenever necessary for the proper selection of the transmitter locations. Transmitter powers are set according to the intended viewer population.

\section{Processing of Study Files}

After the specification of the transmitter, the results of electromagnetic field simulations are obtained as study files, which contain field strengths as $\mathrm{dB} \mu V / m$, over radial lines centered at the transmitting locations. For TV planning, simulations are performed at frequencies that are chosen as representatives for each band. Angular and radial resolution of these study files vary for different transmitting sites depending on demographic requirements. Performing the electromagnetic simulation studies with the terrain information is the most time consuming part of the planning.

It must be noted that it is sufficient to obtain these study files for one transmission power only. For powers other than the simulated value, the field strengths are easily computed as

$$
\Delta F=\Delta P
$$

where $\Delta P$, and $\Delta F$ stand for $\mathrm{dB}$ changes in the transmission power and the field strengths, respectively. The simulations are performed using omnidirectional antennas, and angular dependences of the emitted RF power from the actual planned antennas are easily incorporated similarly.

In storing these study files, field strengths at the simulation points are hardlimited between the values -128 and $+127 \mathrm{~dB}$, and quantized to integers, so that they can be stored in byte sized variables. The large number of transmitter sites makes such a data reduction necessary. Using these files, the field strengths at arbitrary coordinates are obtained by interpolation.

In this project about $1000 \mathrm{TV}$ transmitting sites are planned, whose study files required a storage space of approximately 0.6 


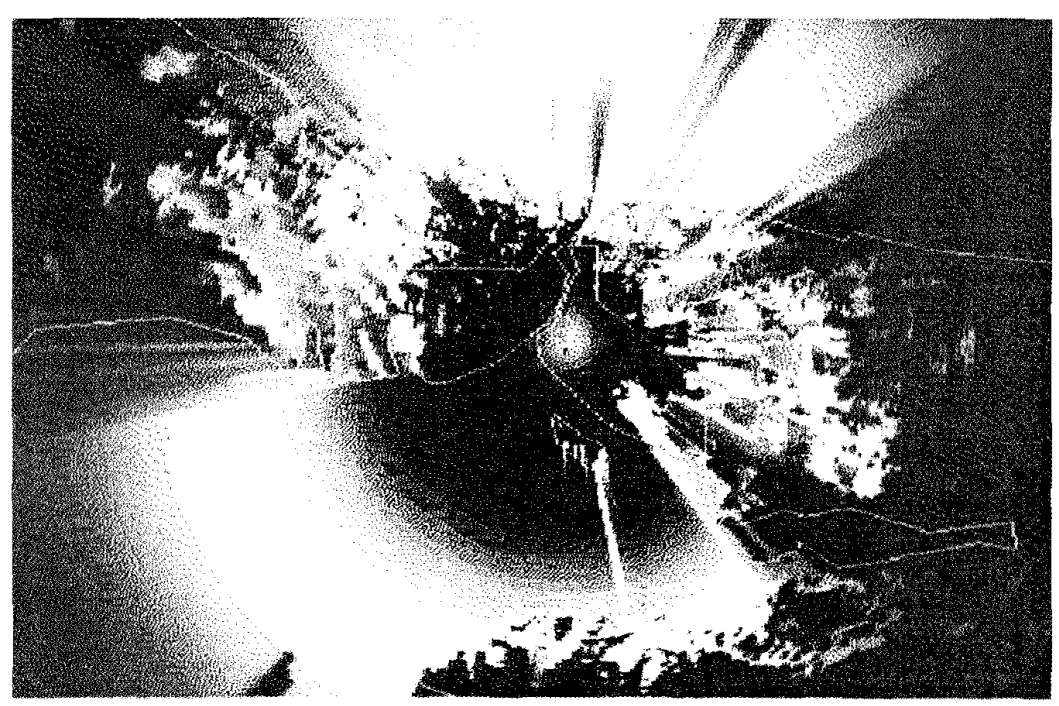

Figure 3. Gray scale plotting of the field strength around Camlica (Istanbul) station in the VHF. Geographical land-sea borders and the metropolitan area are shown by white contours. For illustration purposes increasing levels of ERP is shown by alternating black and white colors.

Gigabytes after such a reduction described above.

\section{Interference Graph}

The interference graph $G(\alpha)$ for a given protection ratio $\alpha$ is a connectivity graph of transmitters over a region. Two transmitters on this graph are connected if one of them broadcasts strong enough signals to cause interference in the coverage area of the other. This connection is called an edge. The direction of the edge is from the interferer to the interfered transmitter. Fig. (4) illustrates the determination of the existence of an edge between the two transmitters $i$ and $j$. Let the coverage area of transmitter $j$ is the elliptic region enclosed by the solid elliptic curve. Let the capital letters $A, B, \ldots$ denote populated sites (sites with a population over a minimum) in this coverage area. Let $F_{i}\left(\vec{x}_{A}\right)$ denote the field strength in decibels, at a populated site A located at world coordinate $\vec{x}_{A}$, that is caused by the $i^{\text {th }}$ transmitter. An edge $E_{i j}$ from node $i$ to node $j$ is introduced if transmitter $i$ interferes with transmitter $j$. So, an edge $E_{i j}$ indicates that there exist at least one populated point $\vec{x}_{p}(\mathrm{P}=\mathrm{A}, \mathrm{B}, \ldots)$ in the coverage area of the $j^{\text {th }}$ transmitter such that

$$
\delta_{i j}=F_{j}\left(\vec{x}_{A}\right)-F_{i}\left(\vec{x}_{A}\right)<\alpha
$$

where $\alpha$ is the protection ratio and $\delta_{i j}$ is related to the quality of the signal received by a receiver located at site A. Hence, if $\alpha$ is the co-channel protection ratio and in the interference graph $G(\alpha)$ there is an edge between two nodes, the same channel should not be used on these two nodes. Significance of a world coordinate $\vec{x}$ is determined by using demographic information about that point, e.g. the population around that point, the proximity of that point to a road, lake, or a military area etc.. Mountain tops are the points that interference occurs most likely. Fortunately, they usually are not demographically critical. The interference graph is generated for various protection ratios using the study files and a demographic database of Turkey.

Repeating the same channel is highly desirable for efficient utilization of the spectrum. For TV broadcasts, it is possible to lower the co-channel protection ratio and reduce the number of edges in the interference graph by using frequency offset between transmitters. For practical reasons, three nonprecision offset values $(8 \mathrm{M}, \mathrm{O}, 8 \mathrm{P})$ are used. If different offsets can be assigned to two different transmitting sites, the co-channel protection ratio drops by $12 \mathrm{~dB}$.

Using the study files, the interference graph is generated for two values of co-channel protection ratio $\alpha_{1}, \alpha_{2}$, corresponding to co-channel protection values with and without offsets, respectively. Naturally $\alpha_{1}<\alpha_{2}$, and, if there exists an edge $E_{i j}$ in $G\left(\alpha_{1}\right)$ the same edge exists also in $G\left(\alpha_{2}\right)$, but the converse does not necessarily hold. Let $\mathcal{E}$ denote the set of edges that exist in $G\left(\alpha_{2}\right)$ but not in $G\left(\alpha_{1}\right)$. After assigning offsets to transmitters, the resultant interference graph $\mathcal{G}$ is formed by adding edges $E_{i j} \in \mathcal{E}$ to $G\left(\alpha_{1}\right)$ under the condition that the same offset is assigned to $i^{\text {th }}$ and $j^{\text {th }}$ transmitters. Let $M(\mathcal{G})$ define the total number of edges in graph $\mathcal{G}$. The offsets must be assigned such that $M(\mathcal{G})$ is as small as possible. There are also some constraints in assigning offsets to transmitters. These constraints are determined by rights and restrictions that are specified by international agreements (Stockholm 1961) and by specifications of some of the transmitters that are already operational in Turkey.

The task of minimizing $M(\mathcal{G})$ under the above mentioned constraints by assigning offsets to transmitters is a combinatorial optimization problem. This problem belongs to the class of NP. complete problems and there is no known algorithm that can find the exact minimizing offset combination for 1000 transmitters in 


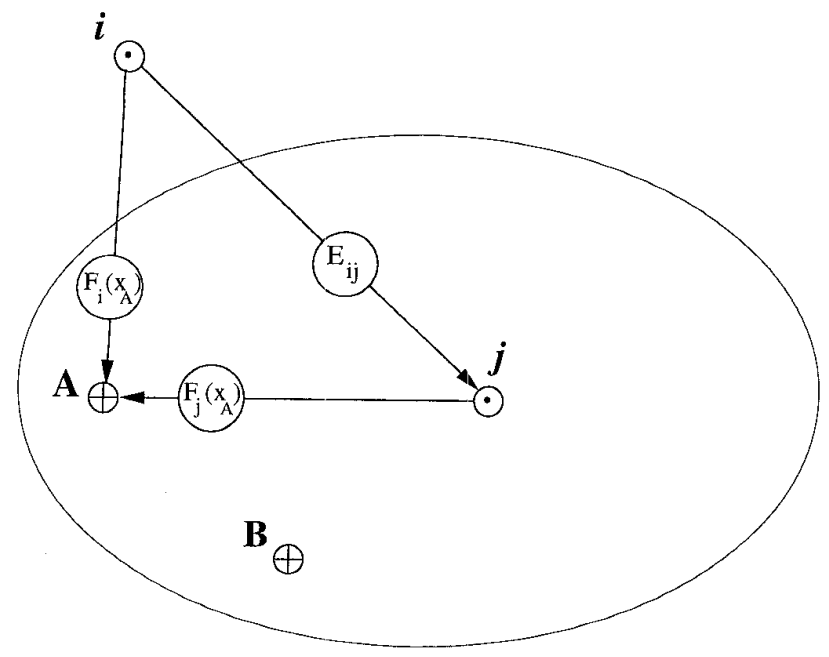

Figure 4. The Interference graph $G(\alpha)$ which is used to determine the existence of an edge between two transmtters $i$ and $j$.

a reasonable time. Observe that the total search space consists of $3^{1000}$ combinations. Simulated Annealing is employed for finding acceptable solutions to this problem. There is a vast amount of literature on the method of Simulated Annealing [7], so we suffice to present its application to the offset assignment problem.

\section{Offset Assignment by Using Simulated Annealing}

Initially, a random offset combination which complies with the constraints is generated and $M(\mathcal{G})$ is evaluated. Then the following steps are repeated for a gradually decreasing parameter $T$ (temperature) until it reaches a final predetermined value.

i) Choose a transmitter randomly and generate a random offset value for that transmitter that complies with the constraints.

ii) Compute the change in cost function

$$
\Delta M(\mathcal{G})=M\left(\mathcal{G}_{\text {new }}\right)-M\left(\mathcal{G}_{\text {old }}\right)
$$

iii) Accept the new configuration with the probability $P$ computed as

$$
P= \begin{cases}1 & \text { if } \Delta M(\mathcal{G}) \leq 0 \\ \exp \left(\frac{-\Delta M(\mathcal{G})}{T}\right) & \text { if } \Delta M(\mathcal{G})>0\end{cases}
$$

The above algorithm generates a sequence of configurations with statistically decreasing costs.

Observe that during iterations a new configuration is immediately accepted if it decreases the cost, otherwise it is accepted with a probability that decreases exponentially by an increase in the cost. It is this nonzero probability that enables the algorithm to escape from the local minima. As $T$ decreases, it becomes less likely for the algorithm to accept a configuration that increases the cost. The process of decreasing $T$ over iterations is called "cooling schedule" in the Simulated anealing terminology. De creasing the temperature too fast may give rise the algorithm to be stuck at a local minimum that is too far from the absolute minimum. The algorithm on the other hand takes a long time if the temperature is decreased too slowly.

Various methods are proposed in the literature for determin ing the initial value of $T$ and the cooling schedule [8]. Simulated Annealing takes a reasonable amount of time (a few minutes compared to several hours for finding $G(\alpha)$ and weeks to complete the electromagnetic simulations in a Sparc 20). Hence, the easiest way of determining the cooling schedule and the initialvalue of $T$ is trial and error, and monitoring the value of the cost function versus $T$ over the iterations. The algorithm eventually reaches a statistical steady state if the iterations are performed at a fixed $T$. A good idea is to decrease the temperature just before the statistical steady state is reached. If the temperature is decreased sufficiently slowly then the final cost should be very close to the minimum cost that occurs until then, and different runs with the same cooling schedule should result in very close optimal values as the solution.

The final (nearly optimal) offset assignment yields the desired interference graph with minimal number of edges. The resultant interference graph is then passed to another program that assigns channels to transmitting sites.

\section{Channel Assignment For Transmitters}

A program has been developed for channel assignment for all transmitters in a network. This program uses three different kinds of input data. One of them is the interference graph. The second type of data fed to the program is a list of forbidden channels for each transmitter. Due to the international agreements like Stockholm 1961 Plan, some channels cannot be used in the transmitters throughout the border area of a country. For this reason, the program assigns channels from a set of allowed channels to each transmitter. The third type of data used by the program is the demographic database including the population of cities, towns, and villages. By means of this database, the program assigns more channels to those transmitters whose coverage area includes bigger population.

Using these input data, firstly, the program tries to assign a minimum number of channels to every transmitter in the network. This minimum number of channel is given by the user. Then, the program starts to assign additional channels to each transmitter by increasing the number of channels one by one. At this point, if there are several alternatives, $a$ new channel is assigned to that transmitter whose coverage area has the biggest population.

\section{Generated Outputs}

The frequency plan is a list of transmitter sites with geographic and technical properties. The geographical properties consist of the coordinates, the height above the sea level, and the antenna height. The technical properties consist of the transmit- 
ter power, maximum effective radiated power in all directions, assigned frequency, polarization, offset, and the vertical tilt angle. For the Turkish TV network, a total of 21652 frequencies at 880 transmitter sites are listed. For the FM Radio network, a total of 30629 frequencies at 800 transmitter sites are listed.

In addition to the plan, two databases are generated for licensing purposes. In the first one, for each transmitting station a list of places down to smallest inhabited districts in the coverage area are given together with total population. For each provincial district, a list of transmitting stations and channels that can be viewed are listed in the second one. Various statistics like average number of channels per transmitting site that can be viewed, etc. can be extracted from those databases.

\section{Conclusions}

A computer aided frequency planning is presented for the VHF and UHF broadcasts. The approach has utilized the Simulated Annealing algorithm for the optimization of number of broadcast channels. It is seen that, this has resulted in a very fast and efficient way of channel assignment. The approach can be generalized to similar mobile and fixed networks.

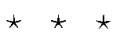

Part of this paper has been presented in NATO-AGARD

Meeting, "Digital Communications Systems: propagation effects, technical solutions, systems design", Sept.1995, Athens, Greece.

\section{References}

[1] A. Quellmalz, A. Knällmann, B. Müller, "Efficient Frequency Assignment with Simulated Annealing" IEE ICAP'95, Eindhoven, The Netherlands, 4-7 April 1995.

[2] R. Grosskopf, "Comparison of Field-Strength Prediction Methods in UHF Range", EBU Review-Technical, No.229, June 1988.

[3] "VHF and UHF propagation curves for the frequency range from 30 to $1000 \mathrm{MHz}$ - Broadcasting services", CCIR Recommmendation 370-5, Vol.5, XVIth Plenary Assembly, Dubrovnik 1986.

[4] "VHF/FM planning parameters and methods", Tech.3236E, European Broadcasting Union, Technical Centre, Brussels, July 1982 .

[5] "Planning parameters and methods for terrestrial television broadcasting in the VHF/UHF bands", Tech.3254-E, European Broadcasting Union, Technical Centre, Brussels, May 1988

[6] R. Grosskopf, "Prediction of Urban Propagation Loss", IEEE Trans. Antennas and Propagation, Vol.42, No.5, May 1994.

[7] E.H.L. Aarts, and P.J.M. van Laarhoven, Simulated Annealing: Theory and Applications, Reidel, Dordrecht, 1987.

[8] B. Hajek, "Cooling Schedules for Optimal Annealing", Mathematics of Operations Research, Vol.13, 1988. 